\title{
Listening to membrane potential: photoacoustic voltage-sensitive dye recording
}

Haichong K. Zhang

Ping Yan

Jeeun Kang

Diane S. Abou

Hanh N. D. Le

Abhinav K. Jha

Daniel L. J. Thorek

Jin U. Kang

Arman Rahmim

Dean F. Wong

Emad M. Boctor

Leslie M. Loew 


\title{
Listening to membrane potential: photoacoustic voltage-sensitive dye recording
}

\author{
Haichong K. Zhang, ${ }^{a, \dagger}$ Ping Yan, ${ }^{b, \dagger}$ Jeeun Kang, ${ }^{a}$ Diane S. Abou, ${ }^{c}$ Hanh N. D. Le, ${ }^{d}$ Abhinav K. Jha, ${ }^{c}$ \\ Daniel L. J. Thorek, ${ }^{c, e}$ Jin U. Kang, ${ }^{d}$ Arman Rahmim, ${ }^{c, e}$ Dean F. Wong, ${ }^{c, f, g, h}$ Emad M. Boctor, ${ }^{a, c, d, \star}$ and \\ Leslie M. Loew ${ }^{b, *}$ \\ a Johns Hopkins University, Department of Computer Science, Baltimore, Maryland, United States \\ bUniversity of Connecticut School of Medicine, R. D. Berlin Center for Cell Analysis and Modeling, Farmington, Connecticut, United States \\ 'Johns Hopkins University School of Medicine, Russell H. Morgan Department of Radiology, Baltimore, Maryland, United States \\ dJohns Hopkins University, Department of Electrical and Computer Engineering, Baltimore, Maryland, United States \\ e Johns Hopkins University School of Medicine, Sidney Kimmel Comprehensive Cancer Center, Department of Oncology, Baltimore, \\ Maryland, United States \\ fJohns Hopkins University, Department of Neuroscience, Baltimore, Maryland, United States \\ gJohns Hopkins University, Department of Psychiatry and Behavioral Sciences, Baltimore, Maryland, United States \\ hJohns Hopkins University, Department of Neurology, Baltimore, Maryland, United States
}

\begin{abstract}
Voltage-sensitive dyes (VSDs) are designed to monitor membrane potential by detecting fluorescence changes in response to neuronal or muscle electrical activity. However, fluorescence imaging is limited by depth of penetration and high scattering losses, which leads to low sensitivity in vivo systems for external detection. By contrast, photoacoustic (PA) imaging, an emerging modality, is capable of deep tissue, noninvasive imaging by combining near-infrared light excitation and ultrasound detection. Here, we show that voltagedependent quenching of dye fluorescence leads to a reciprocal enhancement of PA intensity. We synthesized a near-infrared photoacoustic VSD (PA-VSD), whose PA intensity change is sensitive to membrane potential. In the polarized state, this cyanine-based probe enhances PA intensity while decreasing fluorescence output in a lipid vesicle membrane model. A theoretical model accounts for how the experimental PA intensity change depends on fluorescence and absorbance properties of the dye. These results not only demonstrate PA voltage sensing but also emphasize the interplay of both fluorescence and absorbance properties in the design of optimized PA probes. Together, our results demonstrate PA sensing as a potential new modality for recording and external imaging of electrophysiological and neurochemical events in the brain. $\odot 2017$ Society of Photo-Optical Instrumentation Engineers (SPIE) [DOI: 10.1117/1.JBO.22.4.045006]
\end{abstract}

Keywords: photoacoustics; voltage sensitive dye; fluorescence quenching; brain imaging.

Paper 160821R received Dec. 1, 2016; accepted for publication Mar. 17, 2017; published online Apr. $10,2017$.

\section{Introduction}

The quantification of neurotransmitter (NT) activity with high temporal resolution is essential to build a comprehensive map of brain function. We need to improve upon the low temporal resolution, but high pharmacological specificity, of PET and higher spatial and temporal resolution, but less specific, MRI. Membrane potential measurements, utilizing direct electrical recording or by imaging using voltage-sensitive dyes (VSDs), have been used to observe spontaneous NT events by means of voltage fluctuations caused by ionic currents. Imaging approaches have the advantage, in general, that patterns of activity can be studied with high resolution over large areas of brain. ${ }^{1}$ However, purely optical based detection approaches have several limitations, which include a small dynamic range and only shallow penetration depth due to light scattering and absorbance of overlying tissue in vivo.

Photoacoustic (PA) imaging is an emerging hybrid imaging modality. Here, a noninvasive molecular light absorbancedependent acoustic signal occurs at depths of up to several centimeters in biological tissue, ${ }^{2,3}$ with a micro to millimeter spatial resolution that is limited by the acoustic bandwidth

*Address all correspondence to: Emad M. Boctor, E-mail: eboctor1@jhmi.edu; Leslie M. Loew, E-mail: les@uchc.edu

†These authors have equal contribution. and focusing. The mechanism behind PA imaging is that upon excitation by a short-pulsed laser, thermal relaxation of the chromophore excited state induces local thermal elastic expansion. Using near-infrared laser excitation and ultrasound detection enhances the light-penetration and resulting imaging depth by minimizing the absorptive and scattering attenuation during the light propagation through the biological tissue.

Toward use of this technique for functional brain imaging, previous research has demonstrated PA imaging to be capable of monitoring brain activity based on the blood-oxygen-level dependent signal change. This imaging procedure, which does not rely on the administration of an exogenous contrast agent, ${ }^{4-6}$ is susceptible to several nonlinear physiological and biophysical parameters in addition to the NT activity or electrical signaling. As a result, it is regarded as an indirect, semiquantitative reflection of the membrane potential change in neurons. ${ }^{7,8}$ An alternative approach is needed in order to provide direct readout of membrane potential events in cerebral tissues.

A number of contrast agents have been previously evaluated for use with PA imaging to selectively visualize tumor tissue or metabolic properties. ${ }^{9-11}$ Most of the proposed PA contrast agents have been based on the extinction coefficient of the compound used, as the materials with a stronger absorbance should provide strong PA intensity. Here, we show how

$1083-3668 / 2017 / \$ 25.00$ @ 2017 SPIE 
voltage-dependent PA signals may be produced by VSDs. We first develop the theoretical concept whereby the fluorescence quenching of the voltage-dependent dye leads to a reciprocal enhancement of PA intensity. Based on this concept, we synthesized a near-infrared PA-VSD (PAVSD800-2), whose PA intensity change is sensitive to membrane potential. The performance of the PA-VSD developed was tested with a lipid vesicle test system that allowed us to readily manipulate membrane potential and measure both the PA and spectrophoto/ fluorometric response. Importantly, it has near-infrared excitation and emission bands, which would make it appropriate for deep NT activity imaging applications. Furthermore, our theoretical model based on the photophysical properties of the VSD enables us to quantitatively estimate the PA voltage sensitivity. Further development of the ideas described herein promises exogenous contrast agents with high temporal and spatial resolution for deep brain NT activity measurements.

\section{Theory and Principle}

\subsection{Design of a Photoacoustic Voltage-Sensitive Dye Based on Photophysics and Photochemistry}

When a chromophore absorbs a photon to occupy an excited state, it can relax back to the ground state either by emitting a photon or by shedding its energy as heat. The former is termed "radiative decay" and has a rate, $k_{\mathrm{r}}$, that depends on the chromophore and its molecular environment; the radiative decay is measured as fluorescence. The thermal decay route, with a rate $k_{\mathrm{t}}$, also depends on both the structure of the dye molecule and its environment. Specifically, low energy internal vibrational modes can facilitate thermal relaxation; and the environment, including interactions with solvent or with specific interacting partners - quenchers-also offer nonradiative decay pathways. The PA intensity depends on the thermal decay of chromophores after they are excited by a short intense laser pulse; the resultant rapid and large thermal decay produces a burst of heat that locally increases the kinetic energy of neighboring molecules and can be detected with an ultrasound detector. The key point is that for a given chromophore, the efficiency of thermal $(t h)$ (acoustic) and radiative [fluorescence $(F)$ and phosphorescence] decay processes are in competition; for most organic chromophores, phosphorescence can be neglected, leading to simple relationships:

$\Phi_{F}=\frac{k_{\mathrm{r}}}{k_{\mathrm{r}}+k_{\mathrm{t}}}, \quad \Phi_{t h}=\frac{k_{\mathrm{t}}}{k_{\mathrm{r}}+k_{\mathrm{t}}}$.

These equations give the theoretical quantum efficiency, $\Phi$, for fluorescence and PAs, corresponding, respectively, to the probability that an absorbed photon will be transformed into an emitted photon, detectable as fluorescence, or into a thermoelastic expansion of an absorber, detectable as PA. Both arithmetically and by the principle of conservation of energy, the sum of $\Phi_{F}$ and $\Phi_{t h}$ must be unity. Indeed, there is experimental evidence of the reciprocal relationship between fluorescence efficiency and PA efficiency. For example, Qin et al. ${ }^{12}$ designed a PA contrast agent, in which PA intensity was enhanced by suppressing the fluorescence emission.

It has long been known that cyanine dyes have a tendency to form aggregates at high concentrations. The aggregates are nonfluorescent. Waggoner et al. developed a series of highly sensitive VSDs based on cyanine dyes that, because they have delocalized positive charge, redistributed across cell membranes as a function of the membrane potential. ${ }^{13}$ Because the charge is delocalized, they are able to permeate through the hydrophobic cell membrane and redistribute according to the Nernst equation. The idea is that the dye molecules will accumulate inside polarized cells at sufficiently high local concentration to produce nonfluorescent aggregates; upon depolarization, the dye molecules will be released and diluted into the larger external volume, favoring fluorescent monomers (Fig. 1). Indeed, under the right circumstances, depolarization can produce $>100$ fold increases in cyanine dye fluorescence, ${ }^{14}$ because the tendency for the dyes to aggregate shifts the equilibrium for more monomeric dye to be driven in by the membrane voltage.

We reasoned in the present work that there should be a reciprocal relationship between the fluorescence and the PA sensitivity to membrane potential (Fig. 1) because the aggregated state of the cyanine dyes should favor thermal decay of the excited state. However, previously reported cyanine dyes with superior voltage sensitivity, such as di-SC2(5), ${ }^{14}$ absorb around $650 \mathrm{~nm}$

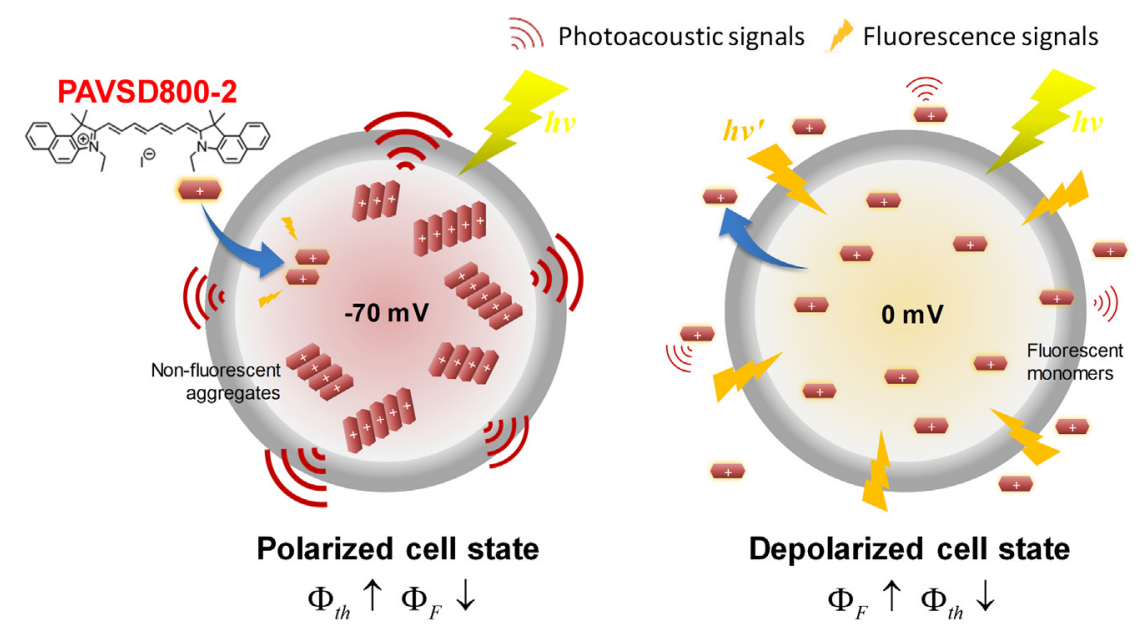

Fig. 1 The principle of the fluorescence quenching effect on the developed VSD (PAVSD800-2) according to a varying membrane potential: typically -70 and $0 \mathrm{mV}$ in the polarized/depolarized states of neurons, respectively. 
or lower. Because the best available laser systems for PA detection are in the near-infrared spectral range and because longer wavelengths allow deeper penetration into tissue, we decided to design and synthesize a new cyanine dye specifically for application as PA-VSD. Our first candidate, PAVSD800-2, shows a fluorescence emission in the near-infrared region with the absorbance peak around $800 \mathrm{~nm}$ and fluorescence emission at $828 \mathrm{~nm}$ in the presence of lipid membranes.

\subsection{Thermal Confinement and Fluorescence Emission}

To be able to quantitatively predict the PA response to a change in membrane potential, we derived a more detailed mathematical model to incorporate the PA signal enhancement with the fluorescence quenching effect. This model also accounts for possible changes in dye absorbance. In the classic formulation, the initial PA pressure, $p_{0}$, has been modeled based on absorbance as

$p_{0}=\frac{\beta}{\rho C_{v} \kappa} \Phi_{t h} \mu_{\mathrm{a}} \Upsilon=\Gamma \Phi_{t h} \mu_{\mathrm{a}} \Upsilon$

where $\beta$ is the thermal compressibility; $\rho$ is the mass density; $C_{v}$ is the heat capacity; $\kappa$ is the isothermal compressibility; $\Phi_{t h}$ is the thermomechanical conversion efficiency, $\mu_{\mathrm{a}}$ is the optical absorbance; $\Upsilon$ is the optical fluency, and $\Gamma$ is the thermodynamic conversion coefficient for PA pressure generation, which is also known as the Grüneisen parameter. However, this formulation is not sufficient to model the PA pressure enhancement due to fluorescence change for the purpose of designing VSD. Our new formulation starts from the energy conservation rule based on quantum yield; the total amount of absorbed energy by an absorber (i.e., $E_{\text {abs }}=\mu_{\mathrm{a}} \Upsilon$ ) will be converted into thermal energy $\left(E_{t h}\right)$, and light re-emission like fluorescence $\left(E_{F}\right)$, and other photochemical reactions $\left(E_{\text {others }}\right)$ :

$E_{\mathrm{abs}}=E_{t h}+E_{F}+E_{\mathrm{others}}$,

and the substitution of Eq. (3) into Eq. (2) gives

$p_{0}=\Gamma E_{t h}=\Gamma\left(E_{\mathrm{abs}}-E_{F}-E_{\mathrm{others}}\right)$.

Now, the PA pressure change ratio (i.e., $C_{\mathrm{PA}}$ ) depending on the neural depolarization can be expressed as

$C_{\mathrm{PA}}=\frac{p_{0}}{p_{0}^{\prime}}=\frac{\left(E_{\mathrm{abs}}-E_{F}\right)}{\left(E_{\mathrm{abs}}^{\prime}-E_{F}^{\prime}\right)}$,

where $p_{0}$ and $p_{0}^{\prime}$ are the initial PA pressures generated by PAVSD at resting and depolarized states of neurons, assuming that $E_{\text {others }}$ is negligible compared to $E_{F} . E_{\text {abs }}^{\prime}$ and $E_{F}^{\prime}$ are the total energy amount of absorbance and fluorescence emission in action state, respectively.

Correspondingly, the total amount of absorbance and fluorescence change depending on the depolarization state of a neuron can be given by

$C_{\mathrm{abs}}=\frac{E_{\mathrm{abs}}}{E_{\mathrm{abs}}^{\prime}}$

and

$C_{F}=\frac{E_{F}}{E_{F}^{\prime}}$.

In addition, the ratio of fluorescence energy compared to total optical absorbance in the resting state, which is the same as the quantum yield $\Phi_{F}^{\prime}$, can be given by

$\Phi_{F}^{\prime}=\frac{E_{F}^{\prime}}{E_{\mathrm{abs}}^{\prime}}=\frac{C_{\mathrm{abs}}}{C_{F}} \Phi_{F}$.

Therefore, the PA pressure change ratio in Eq. (5) can be reformulated as follows:

$C_{\mathrm{PA}} \cong \frac{C_{F}\left(C_{\mathrm{abs}}^{2}-\Phi_{F}\right)}{C_{\mathrm{abs}}\left(C_{F}-C_{\mathrm{abs}} \Phi_{F}\right)}=\frac{C_{\mathrm{abs}}-\Phi_{F}^{\prime} \cdot C_{F}}{1-\Phi_{F}^{\prime}}$.

Hence, the PA signal change in response to neuronal depolarization is determined by the combination of the absorbance and fluorescence changes, as well as the ratio of the fluorescence energy and the absorbance energy in the depolarized state.

\section{Materials and Methods}

\subsection{Synthesis of PAVSD800-2}

PAVSD800-2 was synthesized by modified literature methods (refer to Fig. 2). ${ }^{15,16}$

3-Ethyl-1,1,2-trimethyl-1H-benzo[e]indolium iodide (2). ${ }^{15}$ 1,1,2-Trimethyl-1H-benzo[e]indole $(1,1.0 \mathrm{~g}, 4.78 \mathrm{mmol})$ and iodoethane $(2.8 \mathrm{~g}, 18.0 \mathrm{mmol})$ were dissolved in 5 -mL acetonitrile under nitrogen. The mixture was sealed in a pressure vessel and allowed to react at $130^{\circ} \mathrm{C}$ for $14 \mathrm{~h}$. Upon cooling down to $5^{\circ} \mathrm{C}$, the solids formed were filtered out and washed with acetone to give 2 as gray crystals $(1.12 \mathrm{~g}, 64 \%)$. ${ }^{1} \mathrm{H}$ NMR $\left(400 \mathrm{MHz}, \mathrm{CD}_{3} \mathrm{OD}\right) \delta 1.63(\mathrm{t}, J=7.4 \mathrm{~Hz}, 3 \mathrm{H}), 1.84(\mathrm{~s}, 6 \mathrm{H})$, $4.68(\mathrm{q}, J=7.4 \mathrm{~Hz}, 2 \mathrm{H}), 7.73(\mathrm{t}, J=6.8 \mathrm{~Hz}, 1 \mathrm{H}), 7.81$ $(\mathrm{t}, J=6.8 \mathrm{~Hz}, 1 \mathrm{H}), 8.02(\mathrm{~d}, J=8.8 \mathrm{~Hz}, 1 \mathrm{H}), 8.17(\mathrm{~d}, J=$ $8.4 \mathrm{~Hz}, 1 \mathrm{H}), 8.25(\mathrm{~d}, J=9.2 \mathrm{~Hz}, 1 \mathrm{H}), 8.33(\mathrm{~d}, J=$ $8.4 \mathrm{~Hz}, 1 \mathrm{H}) ; \mathrm{LCMS}: \mathrm{m} / \mathrm{z}=238.0[\mathrm{M}-\mathrm{I}]^{+}$.

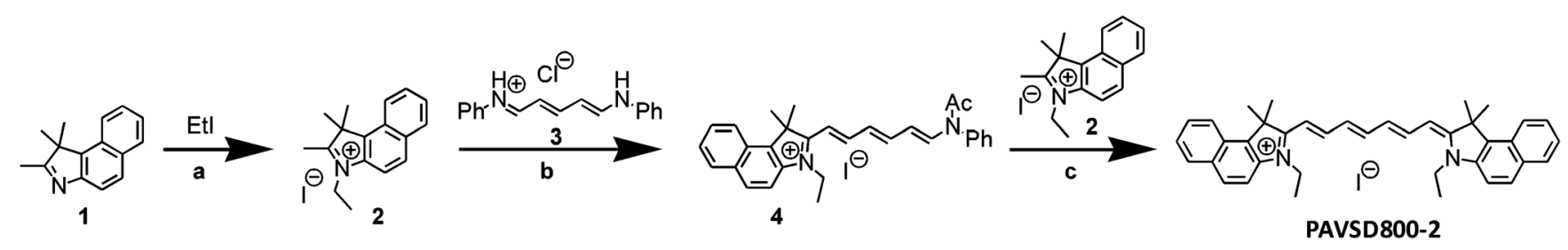

Fig. 2 PAVSD800-2 synthetic scheme. Conditions: (a) $\mathrm{CH}_{3} \mathrm{CN}, 130^{\circ} \mathrm{C}, 14 \mathrm{~h}, 64 \%$; (b) $\mathrm{Ac}_{2} \mathrm{O}, 130^{\circ} \mathrm{C}, 1 \mathrm{~h}$; and (c) $1: 1 \mathrm{Ac}_{2} \mathrm{O} /$ pyridine, $100^{\circ} \mathrm{C}, 40 \%$. 


\subsubsection{Intermediate 4}

Benzoindolium 2 (128 mg, $0.35 \mathrm{mmol})$ and glutaconaldehyde dianil hydrochloride $(3,100 \mathrm{mg}, 0.35 \mathrm{mmol})$ were dissolved in $2 \mathrm{~mL}$ of acetic anhydride, and the mixture was allowed to react at $130^{\circ} \mathrm{C}$ in a pressure vessel for $1 \mathrm{~h}^{16}$ The solution turned purple. The progress of reaction was followed by absorbance spectra in methanol, which showed a major peak at $501 \mathrm{~nm}$ upon completion. This reaction mixture was used for the next step directly.

\subsubsection{Cyanine PAVSD800-2}

A second equivalent of benzoindolium $2(128 \mathrm{mg}, 0.35 \mathrm{mmol})$ and $2 \mathrm{~mL}$ of pyridine were slowly added to the intermediate (4) in acetic anhydride. The mixture was allowed to react at $100^{\circ} \mathrm{C}$ for $30 \mathrm{~min}$, and the solution turned green. The progress of reaction was followed by absorbance spectra in methanol, which showed a major peak at $780 \mathrm{~nm}$ upon completion. After cooling down, the solvents were removed by rotary evaporation and the residue was purified by chromatography $\left(\mathrm{SiO}_{2}\right.$-amino bond, 2:98 $\mathrm{MeOH} / \mathrm{CH}_{2} \mathrm{Cl}_{2}$ ). Green colored fractions were combined and the solvents were removed by rotary evaporation. The residue was washed with isopropanol $(2 \times 10 \mathrm{~mL})$ and then dissolved in $30 \mathrm{~mL} 1: 2 i-\mathrm{PrOH} / \mathrm{CH}_{2} \mathrm{Cl}_{2}$. The solvents were evaporated slowly under vacuum until about $5 \mathrm{~mL}$ was left. Precipitates formed were filtered out and washed with $10 \mathrm{~mL}$ $i$-PrOH, and dried to give cyanine PAVSD800-2 as green powders (94 mg, 40\%). $R_{f}$ (silica gel, $1: 9 \mathrm{MeOH} / \mathrm{CH}_{2} \mathrm{Cl}_{2}$ ) $=0.64$; ${ }^{1} \mathrm{H}$ NMR (400 MHz, CD $\left.\mathrm{OD}\right) \delta 1.42(\mathrm{t}, J=7.2 \mathrm{~Hz}, 6 \mathrm{H}), 1.97$ $(\mathrm{s}, 12 \mathrm{H}), 4.23(\mathrm{q}, J=7.2 \mathrm{~Hz}, 4 \mathrm{H}), 6.32(\mathrm{~d}, J=14 \mathrm{~Hz}, 2 \mathrm{H})$, $6.56(\mathrm{t}, J=12.4 \mathrm{~Hz}, 2 \mathrm{H}), 7.46(\mathrm{t}, J=6.8 \mathrm{~Hz}, 2 \mathrm{H}), 7.55$ $(\mathrm{d}, J=8.8 \mathrm{~Hz}, 2 \mathrm{H}), 7.58-7.68(\mathrm{~m}, 3 \mathrm{H}), 7.95-8.07(\mathrm{~m}, 6 \mathrm{H})$, $8.21(\mathrm{~d}, J=8.8 \mathrm{~Hz}, 2 \mathrm{H}) ; \mathrm{LCMS}: \mathrm{m} / \mathrm{z}=537.0[\mathrm{M}-\mathrm{I}]^{+}$.

\subsection{Lipid Vesicle Preparation}

Lipid vesicles were prepared from 25-mg soybean phosphatidylcholine (type II) suspended in $1 \mathrm{~mL}^{\circ} \mathrm{K}^{+}$buffer, which contains $100 \mathrm{mM} \mathrm{K}_{2} \mathrm{SO}_{4}$ and $20 \mathrm{mM}$ HEPES. This suspension was vortexed for $10 \mathrm{~min}$ and sonicated in bath-type sonicator for $60 \mathrm{~min}$, yielding a translucent vesicle suspension. $\mathrm{A} \mathrm{Na}^{+}$buffer containing $100 \mathrm{mM} \mathrm{Na}_{2} \mathrm{SO}_{4}$ and $20 \mathrm{mM}$ HEPES was prepared. During experiments, $10 \mu \mathrm{L}$ of vesicle suspension was added to $1 \mathrm{~mL}$ of $\mathrm{Na}^{+}$buffer, resulting in an approximately $100: 1 \mathrm{~K}^{+}$ gradient across vesicle membrane. VSD was added to this suspension. When $2.5 \mu \mathrm{L}$ of $10 \mu \mathrm{M}$ valinomycin-a $\mathrm{K}^{+}$specific ionophore-was added, $\mathrm{K}^{+}$ions were transported from inside to outside of vesicle membranes, resulting in a negative membrane potential. This negative potential drives the positively charged VSDs into the vesicles, which causes aggregation of dyes and quenching of fluorescence. Subsequent addition of $2.5 \mu \mathrm{L}$ of $1 \mathrm{mM}$ gramicidin, a nonspecific monovalent cation ionophore, allows $\mathrm{Na}^{+}$cations to move from outside to inside of vesicle membranes to short circuit the membrane potential.

\subsection{Experimental Setup for Photoacoustic Voltage-Sensitive Dyes Characterization}

\subsubsection{Spectroscopic/fluorometric photoacoustic voltage-sensitive dyes characterization}

A combined spectrophoto/fluorometer system (Spectramax i3x, Molecular Devices) was used to measure both fluorescence and absorbance of the PA-VSD with $1,3,6$, and $9 \mu \mathrm{M}$ concentrations. For fluorescence, the measurement was conducted for the spectral range from 750 to $850 \mathrm{~nm}$ at $10-\mathrm{nm}$ increment, and the absorbance was measured in the range from 600 to $900 \mathrm{~nm}$ at 5 -nm increments. Note that 720 -nm wavelength was used for excitation during spectrofluorometry of the PA-VSD.

\subsubsection{Phantom experimental setup for photoacoustic volt- age-sensitive dyes sensing}

The PA sensing system was employed for the characterization of the synthesized PA-VSD (Fig. 3); Q-switched Nd:YAG laser integrated with an optical parametric oscillator (Phocus Inline and MagicPrism, Opotek Inc.) was used for PA signal generation. With the tunability of the laser system, the spectral responses for near-infrared wavelengths (i.e., 700 to $850 \mathrm{~nm}$ at $10-\mathrm{nm}$ interval) were scanned with $20-\mathrm{Hz}$ pulsed repetition frequency and 5-nm pulse duration. The light was delivered through an optical fiber bundle with a 9-mm round shaped output, which was aligned with an ultrasound receiver. The PAVSD in the lipid vesicles suspension was loaded into transparent tubes with 1.27-mm diameter (AAQ04133, Tygon ${ }^{\circledR}$, SaintGobain Corp.), which were located at around $30 \mathrm{~mm}$ depth in the water tank. Note that the temperature of the water was consistently maintained at $22^{\circ} \mathrm{C}$ during the experiments.

A $10-\mathrm{MHz}$ linear ultrasound probe (L14-5/38, Ultrasonix Corp.) was used for PA-VSD sensing in a practical circumstance with a limited bandwidth (i.e., 75\% $-6 \mathrm{~dB}$ fractional bandwidth). Similarly, a hydrophone with wide flat bandwidth from $250 \mathrm{kHz}$ to $20 \mathrm{MHz}$ (HGL-1000, Onda Corp., California) was used to collect single line radio-frequency (RF) PA pressure signal. Twenty sets of PA data were collected, in which 19 of them were collected using the linear array transducer, and the other was through the hydrophone, and the average and standard deviation values were calculated. The PA signals acquired at the depth of target were used to form an averaged RF signal, which is further processed to present PA intensity at each optical wavelength; this permitted an accurate correlation with the

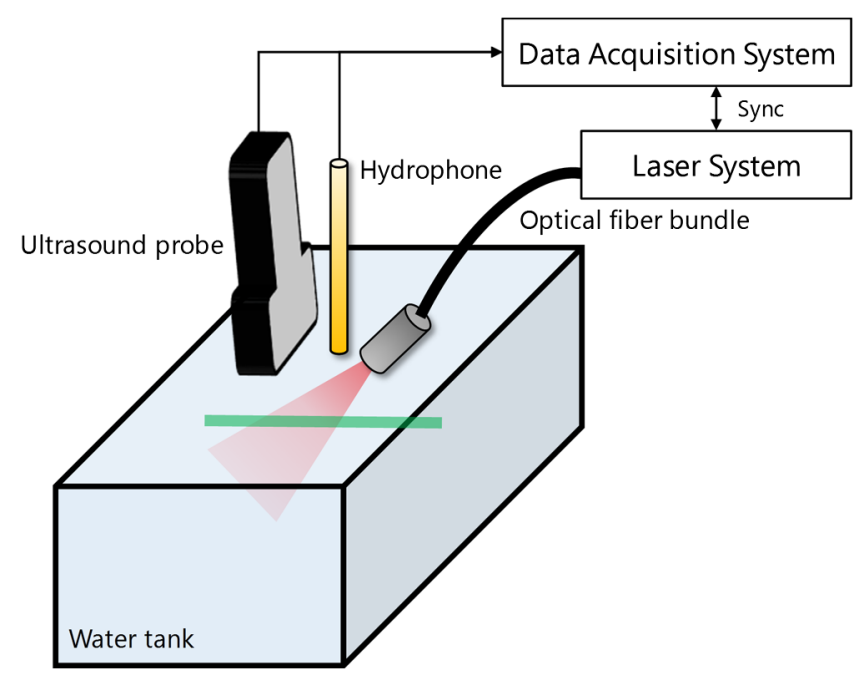

Fig. 3 Phantom experiment setup for PA characterization of PAVSD800-2. Near-infrared light excited the sample in the tubing (green) through the optical fiber bundle, and the generated PA signals were captured by a clinical linear array transducer $(10-\mathrm{MHz}$ center frequency). 
spectrophotometric measurements. For the imaging setup, one set of data was obtained using the linear array transducer from $9 \mu \mathrm{M}$ PA-VSD at 800-nm optical wavelength, and the difference between the resting and polarized states was analyzed.

\section{Results}

\subsection{Spectrophoto/Fluorometric Photoacoustic Voltage-Sensitive Dyes Characterization}

The spectrophoto/fluorometric results of the dye with different concentrations are shown in Figs. 4-6. In the absorbance results, Fig. 4(a) shows the absorbance spectrum for PAVSD800-2 at $6 \mu \mathrm{M}$ concentration, and the peak was determined to be $800 \mathrm{~nm}$. The absorbance slightly decreased in response to the membrane potential at the addition of valinomycin. As the negative control, adding gramicidin into the suspension with valinomycin recovered its absorbance intensity. When the dye concentration of $1,3,6$, and $9 \mu \mathrm{M}$ concentrations were tested, a linear trend of absorbance increase was observed by increasing the dye concentration, and a slight reduction during depolarization is seen for all concentrations [Fig. 4(b)]. A decrease in the primary absorbance peak and an increase in shoulder peak are characteristic of $\mathrm{H}$-aggregation for cyanine dyes. ${ }^{17}$

The measured fluorescence spectra with different PAVSD800-2 concentrations are shown in Figs. 5(a)-5(c). The fluorescence quenching effect is due to negative membrane potential generated by the addition of valinomycin, a $\mathrm{K}^{+}$specific ionophore, and subsequent self-quenching of positive dye molecules accumulating and aggregating inside the vesicles. The effect was reversed when gramicidin, a nonspecific ionophore, was added to depolarize the membrane potential. The spectrum change in fluorescence quenching at $6 \mu \mathrm{M}$ concentration is shown in Fig. 5(a). As negative control, $\mathrm{K}^{+}$buffer was

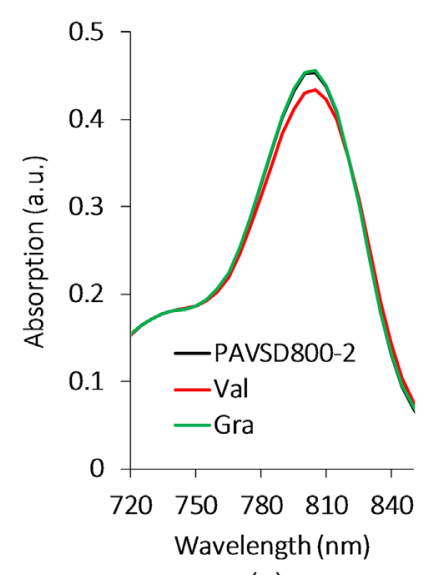

(a)

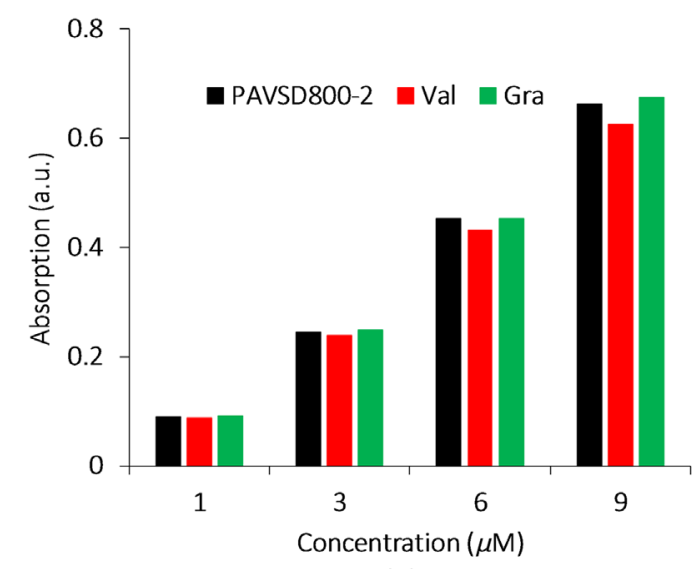

(b)

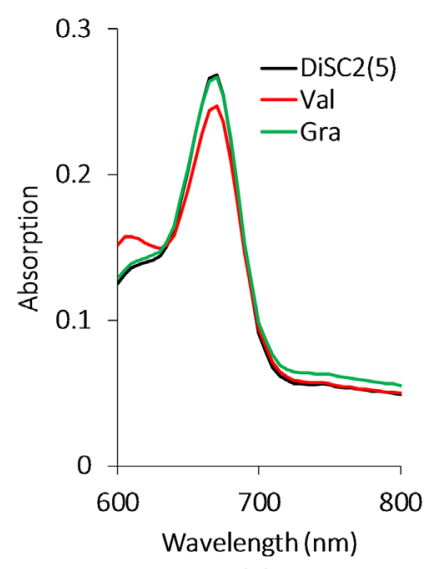

(c)

Fig. 4 The spectrophotometric characteristics of the (a and b) PAVSD800-2 and (c) the di-SC2(5) at the simulated resting/action states using valinomycin (Val) and gramicidin (Gra). (a) Absorbance spectrum of the dye PAVSD800-2 for the concentration of $6 \mu \mathrm{M}$. (b) Absorbance spectra at the wavelength of $800 \mathrm{~nm}$ for different dye concentrations. (c) Absorbance spectrum of the dye di-SC2(5) for the concentration of $6 \mu \mathrm{M}$.

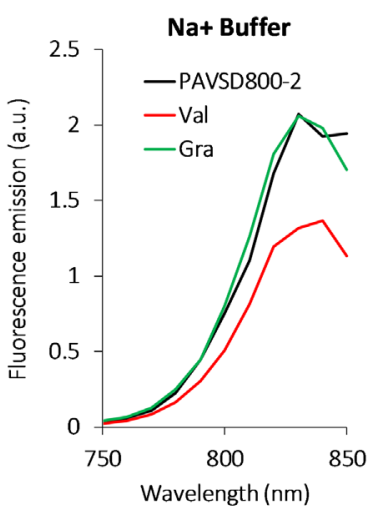

(a)

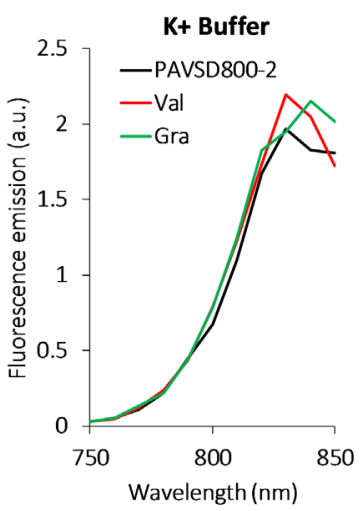

(b)

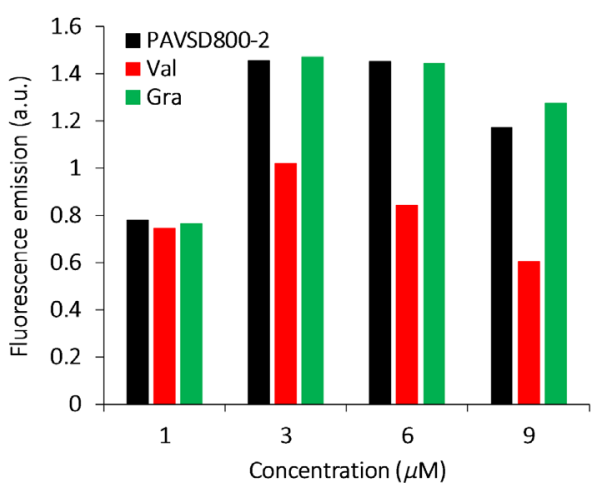

(c)

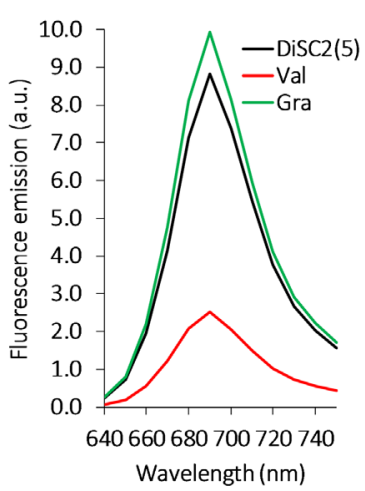

(d)

Fig. 5 The spectrofluorometric characteristics of the (a-c) PAVSD800-2 and (d) the di-SC2(5). (a) The emission spectrum of the dye PAVSD800-2 at $6 \mu \mathrm{M}$ concentration, where vesicles prepared in $\mathrm{K}^{+}$buffer are diluted into isotonic $\mathrm{Na}^{+}$buffer to generate a membrane potential upon addition of valinomycin. (b) Negative control, where $\mathrm{K}^{+}$buffer is used for both the inside and outside. (c) The fluorescence emission at $820 \mathrm{~nm}$ for different concentrations. (d) Fluorescence emission spectrum of the dye, di-SC2(5) at $6 \mu \mathrm{M}$ concentration. 


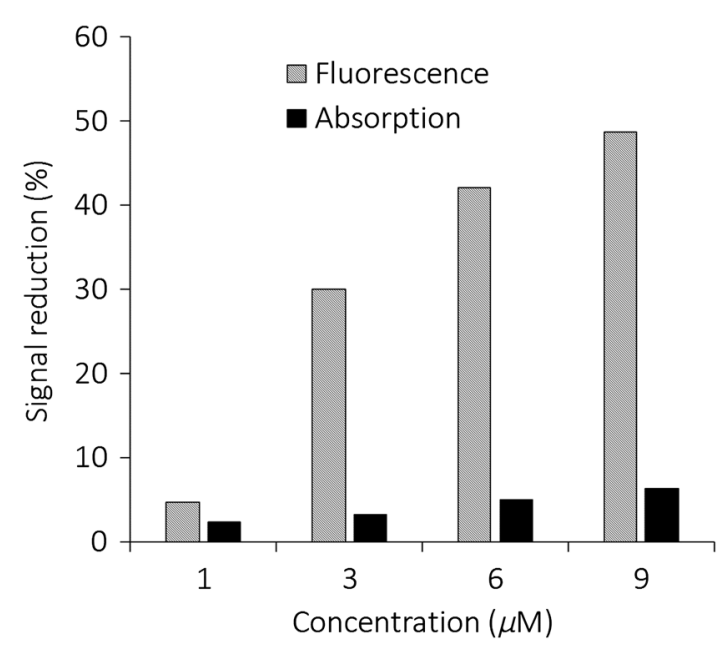

Fig. 6 The fractional change of absorbance at $800 \mathrm{~nm}$ and fluorescence signal at $825 \mathrm{~nm}$ between the resting and action states by adding valinomycin. The fluorescence quenching effect was significantly larger at all concentrations than the absorption change (eight times stronger at $9 \mu \mathrm{M}$ concentration).

used as the external buffer instead of $\mathrm{Na}^{+}$buffer. This control condition establishes the same concentration of $\mathrm{K}^{+}$on both sides of the vesicle membrane, preventing the generation of a polarized voltage when valinomycin is added; the absence of a fluorescence change therefore indicates that the dye does not interact with valinomycin or gramicidin at these concentrations [Fig. 5(b)] but responds only to membrane electrical potential [Fig. 5(a)]. The amount of fractional change in fluorescence emission depends on the dye concentration, and the trend at the emission wavelength of $825 \mathrm{~nm}$ is shown in Fig. 5(c).

A fractional change in absorbance and fluorescence emission for different PAVSD800-2 concentrations are compared and summarized in Fig. 6. A small valinomycin-induced absorbance reduction of $2 \%$ to $6 \%$ were observed from 1 to $9 \mu \mathrm{M}$ concentrations, while the fluorescence quenching up to $49 \%$ was observed for $9 \mu \mathrm{M}$ concentration. This substantial fluorescence change will contribute to the corresponding PA signal sensitivity to membrane potential.

The absorbance and fluorescence spectrum of the classic VSD, di-SC2(5), at the concentration of $6 \mu \mathrm{M}$, are also obtained and shown in Figs. 4(c) and 5(d). This result may be regarded as a positive control to verify that the lipid vesicle system was operating as expected. Figure 5(d) particularly serves to highlight that the magnitude of the fluorescence response can be dramatically large and that improved versions of the longer wavelength cyanine dyes may be achievable.

\subsection{Photoacoustic Characterization of the Photoacoustic Voltage-Sensitive Dyes}

The PA spectrum of $6 \mu \mathrm{M}$ PAVSD800-2 is shown in Fig. 7(a) for a typical experiment. Valinomycin administration, which polarizes the lipid vesicle membranes, increases the PA signal intensity. Addition of gramicidin restores the PA signal intensity close to the original level. In this experiment, the PA intensity at $800 \mathrm{~nm}$ wavelength increases by $14 \%$ in the polarized state. Different concentrations of PAVSD800-2 were also tested at $800 \mathrm{~nm}$ [Fig. 7(b)]. As would be expected, an increase in PA intensity is observed with concentration and the effect of polarization (Val, red) and depolarization (Gra, green) is seen at all concentrations. To confirm the statistical significance of the PA enhancement through polarization, 20 samples were measured, and PA intensity variation was computed by normalizing the intensity to the initial dye intensity. Figure 7(c) shows the mean and the standard deviation of PA intensity variation under three conditions (prestimulus, stimulated polarized, and depolarized states). Comparing the initial depolarized state and after adding valinomycin, and the valinomycin result and the result after adding gramicidin, $p$-values were all below 0.05 . The fractional PA intensity changes upon polarization at 1,3 , 6 , and $9 \mu \mathrm{M}$ concentrations were $17 \%, 24 \%, 17 \%$, and $17 \%$, respectively.

Figure 8 shows the reconstructed PA images with the 800-nm wavelength. A dataset at $9 \mu \mathrm{M}$ concentration, which showed

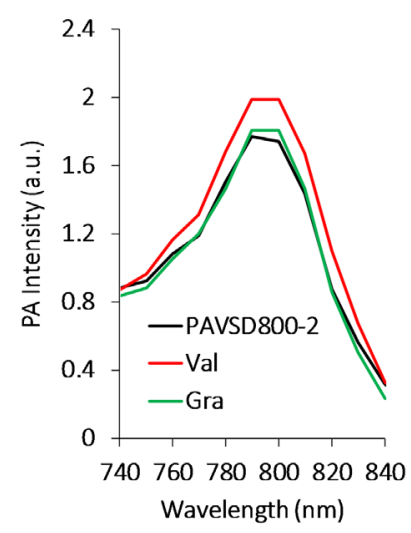

(a)

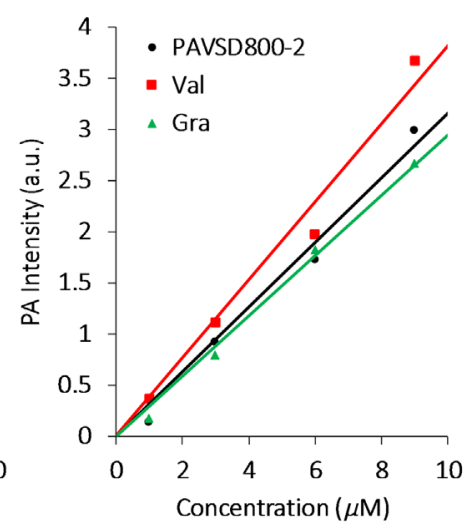

(b)

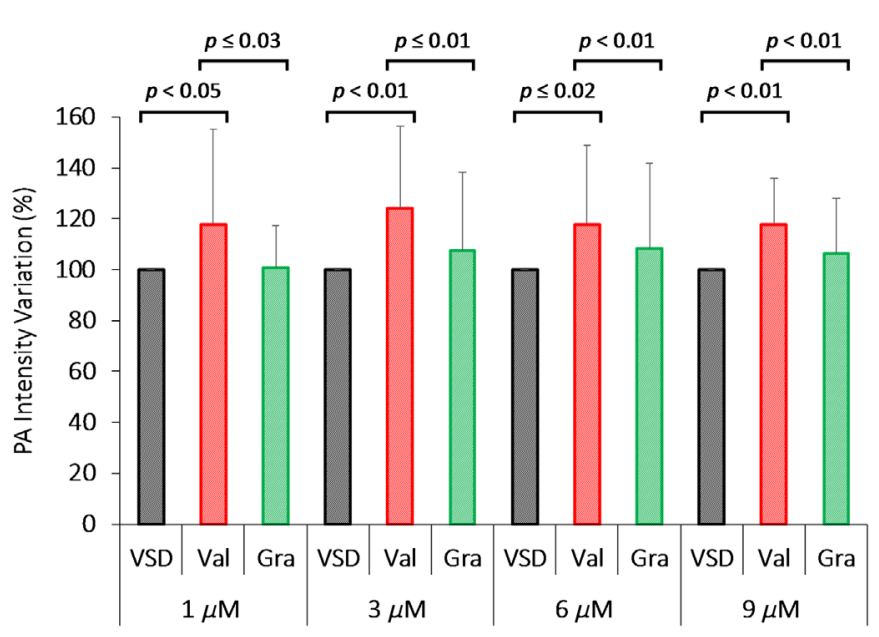

(c)

Fig. 7 (a) PA spectra of the PA-VSD for different concentrations at the prestimulus (black) and stimulated polarized (red) and depolarized (green) states using valinomycin (Val) and gramicidin (Gra). (b) Voltagedependent PA intensity at $800 \mathrm{~nm}$ for different concentrations. (c) PA intensity changes at varying PA-VSD concetrations for polarized (Val, red) and depolarized (Gra, green) states relative to prestimulus (VSD, black). Error bars are standard deviation. 


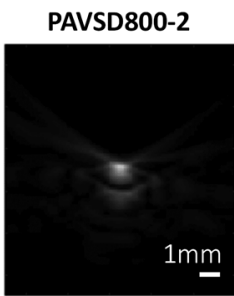

(a)

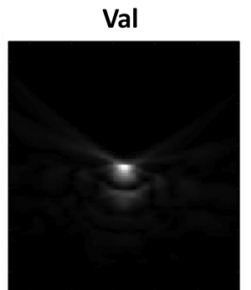

(b)

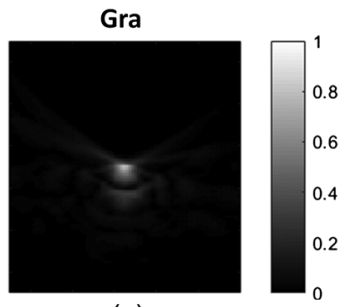

(c)
Fig. 8 The PA images for three conditions: the depolarized state from the (a) PAVSD800-2 only, (b) the valinomycin-induced polarized state, and (c) the gramicidin-reinduced depolarized state.

$22.72 \%$ fractional change of PA signals, was chosen to display. The brighter contrast was observed from the tubing in the valinomycin-induced polarized state, and the contrast was restored to the prestimulus level in the depolarized state after adding the gramicidin.

To further emphasize the change in PA intensity, the before and after valinomycin difference images are shown in Fig. 9. The result after adding gramicidin shows a close intensity to the original data, and it is regarded as the negative control of the evaluation. Note that the same color scale was used on these two results, and a clear enhancement in PA signal intensity was seen at the simulated resting state (i.e., the valinomycininduced negative potential) compared to that of the depolarized state.

\section{Discussion}

In this paper, we report a concept for the design of PA-VSDs based on the fluorescence self-quenching mechanism of cyanine VSDs. We then experimentally validated this idea by designing, synthesizing, and testing a near-infrared absorbing cyanine dye. Based on the results, we demonstrated the promise of PA imaging to detect membrane potential events in real-time.

Our approach relies on the voltage-dependent mechanism of cyanine dye redistribution through cell membranes. It is important to note that this mechanism can be slow on the timescale of seconds. ${ }^{13,14,18}$ This is too slow following the millisecond time scales of neuronal action potentials or electrical events at individual synapses. Studies using two-photon fluorescence imaging microscopy of fast VSDs or genetically encoded voltage indicators can produce high spatial and temporal resolution, $<1 \mathrm{~ms}$ and $<1 \mu \mathrm{m} .{ }^{19-22}$ Although the cyanine dyes are too slow to capture such single spatially localized events, we believe that the proposed VSD technology integrated with PA imaging promises to allow deep monitoring of brain activity patterns on the submillimeter spatial scale and second timescale. Spatial resolution in PA imaging mainly depends on the frequency of the ultrasound receiver and the focusing aperture size. For example, when the receiving ultrasound probe has a center frequency of $10 \mathrm{MHz}$, the maximally expected resolution is $150 \mu \mathrm{m}$ in theory. Potentially, two-photon excitation could permit localized PA detection down to the micrometer scale. Pronounced electrical events, such as seizure patterns, should be readily imaged.
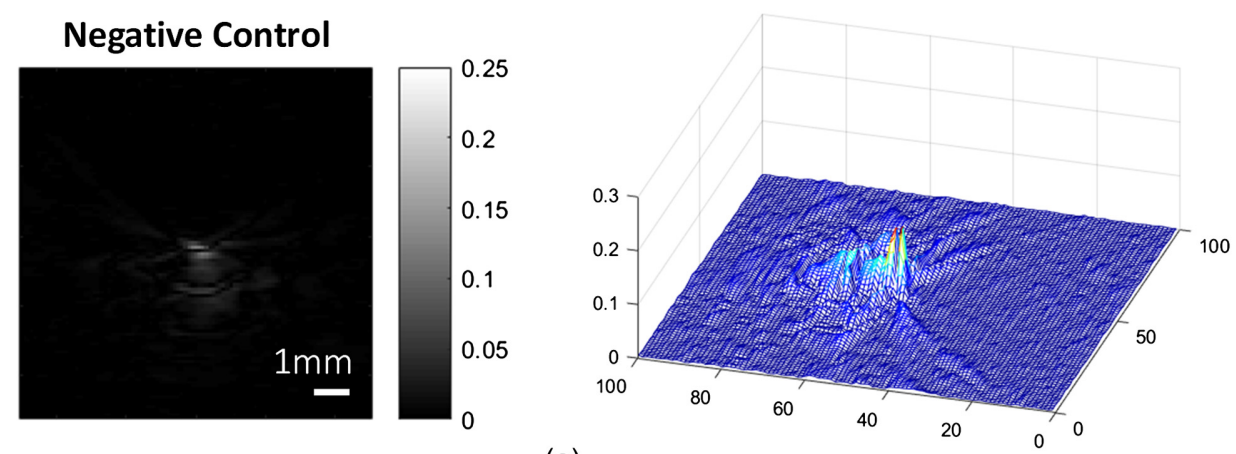

(a)
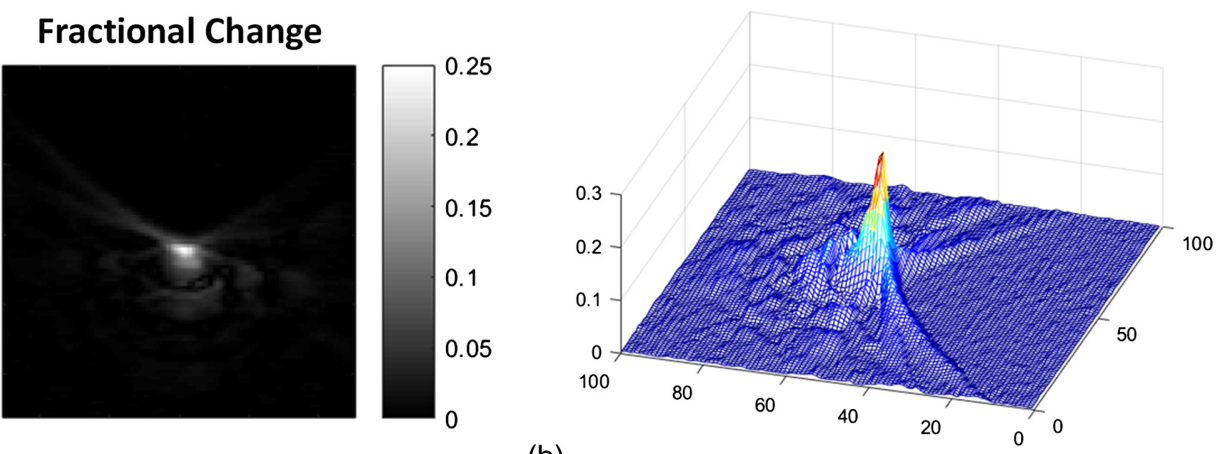

(b)

Fig. 9 Stimulated resting and action state contrast change relative to the prestimulus state intensity. (a) Image produced by subtraction of the gramicidin data from the initial PA dye image is considered a negative control and (b) the difference between the initial PA dye image and the valinomycin-induced polarized state. Magnitude represents the fractional change of the intensity relative to the pre-stimulus state intensity. 
Additionally, because the slow VSDs effectively integrate electrical activity over space and time, spatiotemporal patterns in response to prolonged stimulus and NT release should also be captured. Of course, the studies presented in this report represent the first generation of PA-VSDs with a simple excitation and detection scheme and are intended to demonstrate our design principles. We will use these principles to develop improved PA-VSDs that will capture faster and/or smaller electrical events.

We cannot be certain of the precise membrane polarization induced by valinomycin in our phantom study, because of some inevitable leakage permeability of the lipid vesicle membranes. Nonetheless, the valinomycin-induced membrane polarization has the upper estimate of $-120 \mathrm{mV}$, which is derived from the 100 -fold potassium gradient across the lipid vesicle membranes. From the given polarization, we achieved $24 \%$ and $49 \%$ of fractional changes in PA and fluorescence at, respectively, 3 and $9 \mu \mathrm{M}$ PA-VSD. Therefore, the physiological membrane potential changes in the range of -20 to $-100 \mathrm{mV}$ should be readily detectable. This sensitivity is considerably higher than the best "fast" VSD used for fluorescence monitoring of excitable cells and tissue. ${ }^{22}$ Slow VSD mechanisms can, in general, produce much higher sensitivities than fast VSDs with $\Delta F / F$ as high as $500 \% / 100 \mathrm{mV} ;{ }^{14}$ we therefore anticipate that we will be able to improve the sensitivity of PA-VSDs much further by designing structures with optimized solubility and permeability.

Although the measured absorbance reduction and the fluorescence quenching represent two competing processes to control the magnitude of PA sensitivity, the effect of fluorescence quenching was dominant as observed in our experimental results [compare Figs. 4(a) and 5(a)]. In general, we would need to have measurements of the quantum efficiency $\Phi_{F}$ in each membrane state to be able to make a quantitative prediction of the PA intensity change by applying the theoretical model derived in Eq. (9). Based on this formulation, Fig. 10 shows the PA intensity enhancement for various PAVSD800-2 concentrations over a range of resting $\Phi_{F}^{\prime}$, where the absorbance and the fluorescence change parameters were taken from the experimental measurement results. This graph demonstrates that the PA intensity change is indeed highly sensitive to the fluorescence quantum efficiency. The quenching effect outweighs the absorption

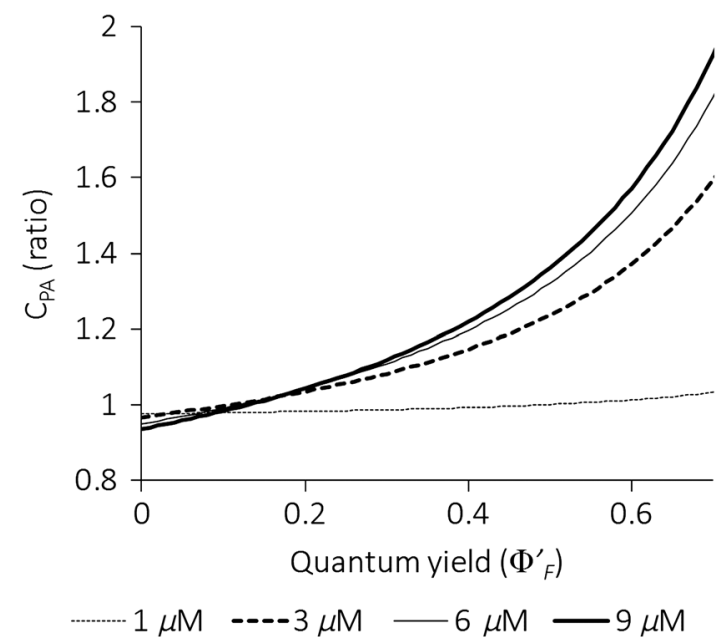

Fig. 10 The theoretical PA signal change based on the absorbance change and fluorescence change from the experimental data. reduction and leads to PA intensity enhancement when the quantum yield is higher than around 0.1 .

The derived theoretical model in Eq. (9) provides a quantitative tool to guide optimization of PA-VSD performance for potential applications in transcranial PA and fluorescence imaging. This model indicates three directions to design a better fluorescence quenching-based VSD: (1) higher fluorescence quenching effect, (2) less absorbance reduction or larger absorbance enhancement upon aggregation, and (3) higher fluorescence efficiency $\Phi_{F}$. It might be counterintuitive that a higher $\Phi_{F}$ would produce a better PA intensity change, but the higher the baseline $\Phi_{F}$ is, the greater the opportunity for large quenching becomes, as more energy transfers into nonradioactive decay. Nevertheless, there should be further optimization between fractional contrast change of PA signals and its sensitivity, which may be lowered by the high $\Phi_{F}$. Our future work will be focused on enhancing the balanced performance of PA-VSDs guided by the theoretical model.

A limitation of the current theoretical model is that the PA or fluorescence change is based on the assumption of a linear system with respect to the energy. However, some studies suggested that the PA intensity could be nonlinearly related to the light energy under certain circumstances. ${ }^{23,24}$ Therefore, the theoretical model can be improved by including potential nonlinear sensitivity of the VSD.

In addition to the dye features that would improve PA voltage sensitivity, other important dye attributes will also be needed to produce practical PA-VSDs for brain imaging. Such improvement might include: even longer wavelengths to further enhance deep tissue imaging; greater aqueous solubility to allow higher concentrations to be delivered to neuronal tissues; dyes with faster voltage-dependent redistribution kinetics to allow for sensing of activity down to the timescale of action potentials or individual NT events. We believe that work described here provides both a theoretical and experimental proof of concept to allow us to further develop PAs as a new modality for imaging electrical activity in the brain.

\section{Conclusions}

In this paper, we presented a PA-based VSD, PAVSD800-2, as a potential tool to monitor membrane potential for brain imaging. The design principle of the dye is to manipulate fluorescence quantum yield to enhance PA intensity through voltage-dependent fluorescence quenching, while the total absorbance remains stable. We also derived the theoretical model to predict the PA intensity change based on known photophysical dye characteristics. This model is experimentally validated, and the reciprocal relationship between PA and fluorescence was demonstrated for different dye concentrations.

\section{Disclosures}

No conflicts of interest, financial or otherwise, are declared by the authors.

\section{Acknowledgments}

Authors thank Mohammad Reza Fouladi for proofreading the manuscript and providing helpful comments. Financial support was provided by NIH Brain Initiative under Grant No. R24MH106083-03 and NIH National Institute of Biomedical Imaging and Bioengineering under Grant No. R01EB01963. 


\section{References}

1. M. Canepari, O. Bernus, and D. Zecevic, Membrane Potential Imaging in the Nervous System and Heart, p. 516, Springer, New York (2015).

2. L. V. Wang and S. Hu, "Photoacoustic tomography: in vivo imaging from organelles to organs," Science 335(6075), 1458-1462 (2012).

3. L. V. Wang, "Multiscale photoacoustic microscopy and computed tomography," Nat. Photonics 3, 503-509 (2009).

4. S. Hu, "Listening to the brain with photoacoustics," IEEE J. Sel. Top. Quantum Electron. 22(3), 117-126 (2016).

5. M. Nasiriavanaki et al., "High-resolution photoacoustic tomography of resting-state functional connectivity in the mouse brain," Proc. Natl. Acad. Sci. U. S. A. 111(1), 21-26 (2014).

6. J. Yao and L. V. Wang, "Photoacoustic brain imaging: from microscopic to macroscopic scales," Neurophotonics 1(1), 011003 (2014).

7. O. J. Arthurs et al., "Intracortically distributed neurovascular coupling relationships within and between human somatosensory cortices," Cereb. Cortex 17(3), 661-668 (2007).

8. O. J. Arthurs and S. Boniface, "How well do we understand the neural origins of the fMRI BOLD signal?" Trends Neurosci. 25(1), 27-31 (2002).

9. J. Weber, P. C. Beard, and S. E. Bohndiek, "Contrast agents for molecular photoacoustic imaging," Nat. Methods 13, 639-650 (2016).

10. D. Wu et al., "Contrast agents for photoacoustic and thermoacoustic imaging: a review," Int. J. Mol. Sci. 15(12), 23616 (2014).

11. G. P. Luke, D. Yeager, and S. Y. Emelianov, "Biomedical applications of photoacoustic imaging with exogenous contrast agents," Ann. Biomed. Eng. 40(2), 422-437 (2012).

12. H. Qin et al., "Fluorescence quenching nanoprobes dedicated to in vivo photoacoustic imaging and high-efficient tumor therapy in deep-seated tissue," Small 11(22), 2675-2686 (2015).
13. P. J. Sims et al., "Studies on the mechanism by which cyanine dyes measure membrane potential in red blood cells and phosphotidylcholine," Biochemistry 13(16), 3315-3330 (1974).

14. L. M. Loew et al., "Fluorometric analysis of transferable membrane pores," Biochemistry 24(9), 2101-2104 (1985).

15. S. H. Sinha et al., "Synthesis and evaluation of carbocyanine dyes as PRMT inhibitors and imaging agents," Eur. J. Med. Chem. 54, 647-659 (2012).

16. R. B. Mujumdar et al., "Cyanine dye labeling reagents: sulfoindocyanine succinimidyl esters," Bioconjugate Chem. 4(2), 105-111 (1993).

17. E. A. McArthur et al., "A study of the binding of cyanine dyes to colloidal quantum dots using spectral signatures of dye aggregation," J. Phys. Chem. C 116(10), 6136-6142 (2012).

18. L. M. Loew, "Design and use of organic voltage sensitive dyes," $A d v$. Exp. Med. Biol. 859, 27-53 (2015).

19. C. D. Acker, P. Yan, and L. M. Loew, "Single-voxel recording of voltage transients in dendritic spines," Biophys. J. 101(2), L11-L13 (2011).

20. C. D. Acker, E. Hoyos, and L. M. Loew, "EPSPs measured in proximal dendritic spines of cortical pyramidal neurons," eNeuro 3(2), 1-13 (2016).

21. A. Walther et al., "Two-photon voltage imaging using a genetically encoded voltage indicator," Sci. Rep. 3, 2231 (2013).

22. P. Yan et al., "A palette of fluorinated voltage sensitive hemicyanine dyes," Proc. Natl. Acad. Sci. U. S. A. 109(50), 20443-20448 (2012).

23. H. Chen and G. Diebold, "Chemical generation of acoustic waves: a giant photoacoustic effect," Science 270(5238), 963-966 (1995).

24. M. Sarimollaoglu et al., "Nonlinear photoacoustic signal amplification from single targets in absorption background," Photoacoustics 2(1), $1-11$ (2014)

Biographies for the authors are not available. 